\title{
NEW DISTRIBUTIONAL DATA ON BRYOPHYTES OF POLAND AND SLOVAKIA, 9
}

\author{
Piotr Górski, Pawet Pawlikowski, Anna Rusińska, Barbara Fojcik, Robert Zubel, \\ Grzegorz Vončina, Henryk Klama, Michą Smoczyk, Anna Salachna
}

\author{
Editors of the column: Piotr Górski, AnNa Rusińska \\ Series "New distributional data on bryophytes \\ of Poland (and Slovakia)" is a scientific bulletin \\ of Bryological Section of Polish Botanical Society

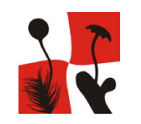

B. Fojcik, Department of Botany and Nature Protection, Faculty of Biology and Environmental Protection, University of Silesia, Jagiellońska 28, 40-032 Katowice, Poland, e-mail: fojcik@us.edu.pl

P. Górski, Department of Botany, Poznań University of Life Sciences, Wojska Polskiego 71 C, 60-625 Poznań, Poland, e-mail: peter@up.poznan.pl

H. Klama, Institute of Environmental Protection and Engineering, University of Bielsko-Biała, Willowa 2, 43-309 Bielsko-Biała, Poland, e-mail: hklama@ath.bielsko.pl

P. Pawlikowski, Department of Plant Ecology and Environmental Conservation, Institute of Botany, Faculty of Biology, Biological and Chemical Research Centre, University of Warsaw, Żwirki i Wigury 101, 02-096 Warsaw, Poland, e-mail: p.pawlikowski@uw.edu.pl

A. Rusińska, Natural History Collections, Adam Mickiewicz University, Umultowska 89, 61-614 Poznań, Poland, e-mail: annarus@amu.edu.pl

A. Salachna, Institute of Environmental Protection and Engineering, University of Bielsko-Biała, Willowa 2, 43-309 Bielsko-Biała, Poland, e-mail: aradzioch@ ath.bielsko.pl

M. Smoczyk, Wojska Polskiego 30/5, 69-110 Rzepin, Poland, e-mail: msmoczyk@wp.pl

G. Vončina, Pieniny National Park, Jagiellońska 107 B, 34-450 Krościenko nad Dunajcem, Poland, e-mail: gvoncina@ poczta.onet.pl

R. Zubel, Department of Botany and Mycology, Maria Curie-Skłodowska University, Akademicka 19, 20-033 Lublin, Poland, e-mail: robert.zubel@poczta.umcs.lublin.pl

AвSTRACt. This work presents a list of localities for the following species: Barbilophozia barbata, Campylopus flexuosus, Conocephalum salebrosum, Didymodon spadiceus, Eucladium verticillatum, Frullania dilatata, Hamatocaulis vernicosus, Harpanthus flotovianus, Pohlia ludwigii, Riccardia latifrons, Scorpidium scorpioides, Sphagnum fuscum, and Tomentypnum nitens.

\section{NEW LOCALITIES}

\section{Barbilophozia barbata (Schmidel ex Schreb.) Loeske}

\section{Author: P. GóRSKI}

SLOVAKIA: High Tatra Mts, MGRS 34UDV2550, NE slope of Mt Velká kopa, above Vyšné Garajovo sedlo pass, $49.20142^{\circ} \mathrm{N}, 19.97749^{\circ} \mathrm{E}$, alt. $1960 \mathrm{~m}$ above sea level (a.s.1.), Polytrichum-Sphagnum hummock, leg., det. P. Górski, 4.08.2015 (POZNB 2022); Poland: ATPOL Gd-59, S Poland, Western Tatra Mts, Polana na Stołach glade, MGRS 34UDV1655, 49.24962 ${ }^{\circ} \mathrm{N}$, $19.85603^{\circ} \mathrm{E}$, alt. $1340 \mathrm{~m}$ a.s.1., calcareous outcrops, leg., det. P. Górski, 20.11.2011 (POZNB 2326); ATPOL Cb-71, W Poland, NW Wielkopolska region, Gorzów Basin, Puszcza Notecka Forest, Lubuskie Province, Międzychód County, Skwierzyna commune, $52.65141^{\circ} \mathrm{N}, 15.86055^{\circ} \mathrm{E}$, ca $2 \mathrm{~km}$ from the village of Radgoszcz, Leucobryo-Pinetum, leg., det. P. Górski, 24.06.2015 (POZNB). 
The liverwort Barbilophozia barbata has scattered populations in northern, central, and southern Poland (SzWEYKOWsKi 1967, 2006). Barbilophozia barbata is relatively common at lower elevations in mountainous regions of Poland. In the Tatra Mountains (GóRSKI \& VÁŇA 2014), most B. barbata populations (89\% of those described) are found in the altitudinal belt, up to $1600 \mathrm{~m}$ above sea level (a.s.l.). Barbilophozia barbata is rarely found at higher altitudes. It has been described at only four localities above $1900 \mathrm{~m}$ a.s.l. (SZWEYKoWSKi 1960, DudA \& VÁŇA 1985).

\section{Campylopus flexuosus (Hedw.) Brid.}

Author: B. FoJCIK

ATMOS Fc-69: S Poland, Silesian Upland (Wyżyna Śląska), Rybnik Plateau (Płaskowyż Rybnicki), Rybnik town, district Popielów, $50.04532878^{\circ} \mathrm{N}, 18.5134528^{\circ} \mathrm{E}$, rotten log in herb vegetation near the edge of deciduous forest, leg., det. B. Fojcik, 7.04.2016 (KTU).

Campylopus flexuosus is a Polish bryoflora species that, although rare, is considered to be spreading; it has been identified at many new sites in recent years (Stebel 2007, 2015, 2016). Campylopus flexuosus occurs mainly in southern Poland, along the Sudeten-Carpathian arc, and only a few populations are located in central and northern Poland (RusińsKA 1995, STEBEL 2007, 2015). The species usually grows in forests on humic and mineral soil. In general, Campylopus flexuosus has a sub-oceanic distribution (DüLl 1984). Campylopus flexuosus is partly protected in Poland and numbered among threatened mosses of undetermined threat (category I, ŻARNOWIEC et al. 2004). It is the first locality of this species from the Silesian Upland.

\section{Conocephalum salebrosum Szweykowski, Buczkow- ska \& Odrzykoski}

Authors: H. Klama, A. Salachna

ATMOS Gd-33: S Poland, Beskid Żywiecko-Orawski Mts, Wielka Racza group (Grupa Wielkiej Raczy), Silesian Province, Zywiec County, Soblówka village, valley of Cichy potok stream, $49.42389^{\circ} \mathrm{N}$, $19.13556^{\circ} \mathrm{E}$, alt. $700 \mathrm{~m}$ a.s.l., on wet soil on the bank of the stream, leg., det. H. Klama, A. Salachna, 23.09.2011, ster. (herb. H. Klama).

Conocephalum salebrosum was first described in 2005 (SzWEYKowski et al. 2005). Thus, all plants collected before 2005 and identified as C. conicum should be reconsidered. Conocephalum salebrosum is a lowland mountain liverwort species and is common in Poland, found throughout the country, mainly in wet places. In the mountains, $C$. salebrosum can grow even above $1000 \mathrm{~m}$ a.s.l. (SZWEYKOWSKI et al. 2005, GóRSKI \& VÁŇA 2014). This thallose plant has been identified in some regions of the Beskidy Zachodnie Mountains (SZWEYKOWSKI et al. 2005, WajDA \& Klama 2014). This is the second locality of $C$. salebrosum in the Beskid Żywiecko-Orawski Mountains (Stebel et al. 2011).

\section{Didymodon spadiceus (Mitt.) Limpr.}

Authors: B. Fojcik, R. Zubel, G. Vončina

ATMOS Gf-09: SE Poland, Eastern Carpathians (Karpaty Wschodnie), Sanocko-Turczańskie Mountains (Góry Sanocko-Turczańskie), Suchy Obycz massive, Kamionka river valley (called Dolina Niemiecka valley), Podkarpackie Province, north of the settlement of Arłamów, $49.61872^{\circ} \mathrm{N}, 22.64158^{\circ} \mathrm{E}$, alt. $427 \mathrm{~m}$ a.s.l., wet stones in the stream in beech forest, leg., det. B. Fojcik, R. Zubel, G. Vončina, 12.09.2015, conf. A. Stebel (KTU, LBL, SOSN).

Didymodon spadiceus is a calcicole and hygrophilous moss that usually grows on base-rich rocks and tree boles adjacent to fast-flowing streams and rivers (DiERSSEN 2001, SMith 2004). In Poland, scattered populations of $D$. spadiceus are mainly found in mountain regions (SZAFran 1957). Didymon spadiceus is classified as a sub-montane species (STEBeL 2006) and has been reported to occur in the Krakowsko-Częstochowska Upland (SZAFRAN 1957). It is the first locality of $D$. spadiceus in the Sanocko-Turczańskie Mountains.

\section{Eucladium verticillatum (Hedw. ex Brid.) Bruch \& Schimp.}

\section{Authors: B. FoJciK, R. Zubel, G. VonČINA}

ATMOS Gf-09: SE Poland, Eastern Carpathians, Sanocko-Turczańskie Mountains, Suchy Obycz massive, Kamionka river valley (called Dolina Niemiecka valley), Podkarpackie Province, north of the settlement of Arłamów, $49.61427^{\circ} \mathrm{N}, 22.641^{\circ} \mathrm{E}$, alt. $433 \mathrm{~m}$ a.s.l., on dripping cliff encrusting with lime deposits (wellhead in beech forest), leg., det. B. Fojcik, R. Zubel, G. Vončina, 12.09.2015, conf. A. Stebel (KTU, LBL, SOSN).

Eucladium verticillatum is a calcicole hygrophilous species that usually grows as dense tufts encrusted with calcareous matter (Dierssen 2001, Smith 2004). Eucladium verticillatum has a sub-mediterranean distribution (DülL 1984) and, in Poland, has only been identified at one southern site (SZAFRAN 1957). Eucladium verticillatum has been reported at the Gorce Mountains (Stebel \& CZarnota 2012), Pieniny Mountains (Stebel et al. 2010) and Krakowsko-Częstochowska Upland (FолсIK 2011), among other sites. It is the first locality of this multizonal mountain species (Stebel 2006) in the Sanocko-Turczańskie Mountains, as well as in the south-eastern part of Poland.

\section{Frullania dilatata (L.) Dumort.}

Author: M. SMOczYK

ATMOS Ca-83: W Poland, Lubuskie Lakeland (Pojezierze Lubuskie), Łagowskie Lakeland (Pojezi- 
erze Łagowskie), $1.9 \mathrm{~km}$ north-west from church in the village of Górzyca, forest section $93 \mathrm{~h}$ of the Ośno Lubuskie Forest Inspectorate, $52.5111^{\circ} \mathrm{N}$, $14.6628^{\circ} \mathrm{E}$, trunk of Robinia pseudacacia in Robinia planting, leg., det. M. Smoczyk, 22.04.2015 (POZG); ATMOS Ca-93: W Poland, Lubuskie Lakeland, Łagowskie Lakeland, $1.4 \mathrm{~km}$ south-west from the church in the village of Sułów, numerous localities in "Mokradła Sułowskie" nature reserve, forest sections 110a, 126k, 141d, 151b, 162a of the Rzepin Forest Inspectorate, on trunk of Alnus glutinosa, Populus sp., Betula pendula, e.g. $52.3759^{\circ} \mathrm{N}, 14.7168^{\circ} \mathrm{E}$, leg., det. M. Smoczyk, 24.10.2015 (POZG); ATMOS Ca-94: W Poland, Lubuskie Lakeland, Łagowskie Lakeland, $2.4 \mathrm{~km}$ south-west from the church in the village of Połęcko, by the forest road from Połęcko to Starościn, forest section 268c of the Rzepin Forest Inspectorate, $52.3893^{\circ} \mathrm{N}, 14.8710^{\circ} \mathrm{E}$, bark of Padus avium, leg., det. M. Smoczyk, 27.04.2013, c. spor. (POZG); ATMOS Da-03: W Poland, Lubuskie Lakeland, Łagowskie Lakeland, $1.9 \mathrm{~km}$ south-west from the church in the village of Sułów, numerous localities in "Mokradła Sułowskie" nature reserve, forest sections 162a, 151b, 151h of the Rzepin Forest Inspectorate, on bark of Fraxinus excelsior, Alnus glutinosa and Betula pendula, e.g. $52.3699^{\circ} \mathrm{N}, 14.7175^{\circ} \mathrm{E}$, leg., det. M. Smoczyk, 12.03.2016 (POZG); ATMOS Da-04: Torzym Plain (Równina Torzymska), 1.6 km west of the Jerzmanice Lubuskie railway station, forest section 530f of the Rzepin Forest Inspectorate, $52.3065^{\circ} \mathrm{N}, 14.8438^{\circ} \mathrm{E}$, on bark of Carpinus betulus, leg., det. M. Smoczyk, 29.06.2015, c. per. (POZG); shore of Jezioro Rzepsko Lake south-east of Rzepin, forest section $423 \mathrm{~g}$ of the Rzepin Forest Inspectorate, $52.3190^{\circ} \mathrm{N}, 14.8831^{\circ} \mathrm{E}$, on bark of Padus avium, leg., det. M. Smoczyk, 21.03.2015 (POZG); Rzepinek settlement, the Ilanka River valley, $1.7 \mathrm{~km}$ south of the church in Rzepin, forest section $491 \mathrm{~b}$ of the Rzepin Forest Inspectorate, thickets where a building formerly sat, $52.3210^{\circ} \mathrm{N}, 14.8227^{\circ} \mathrm{E}$, on a branch of Euonymus europaea, leg., det. M. Smoczyk, 1.04.2014 (POZG); Polęcin settlement north of the village of Starościn, forest section 309b of the Rzepin Forest Inspectorate, $52.3695^{\circ} \mathrm{N}, 14.8598^{\circ} \mathrm{E}$, on bark of $\mathrm{Pa}-$ dus avium by the forest swamp, leg., det. M. Smoczyk, 27.04.2013 (POZG); east of Nowy Młyn settlement, forest section $534 \mathrm{j}$ of the Rzepin Forest Inspectorate, $52.3078^{\circ} \mathrm{N}, 14.8179^{\circ} \mathrm{E}$, on bark of Acer pseudoplatanus in patch of an oak-hornbeam forest from Galio sylvatici-Carpinetum betuli plant association, leg., det. M. Smoczyk, 24.03.2012 (POZG); ATMOS Da-06: W Poland, Lubuskie Lakeland, Łagowskie Lakeland, Prześlicki Bór Forest ca $2.7 \mathrm{~km}$ south-east from the village of Bobrówka, Papiernik former settlement, forest section $51 \mathrm{~h}$ of the Torzym Forest Inspectorate, $52.3513^{\circ} \mathrm{N}, 15.0347^{\circ} \mathrm{E}$, on bark of Crataegus monogyna in thickets in the ruins of a house, leg., det. M. Smoczyk, 16.04.2016 (POZG); ATMOS Da-14:
W Poland, Lubuskie Lakeland, Torzymska Plain, $1.0 \mathrm{~km}$ south-west from the church in the village of Radzików, forest section 1441 of the Cybinka Forest Inspectorate, $52.2606^{\circ} \mathrm{N}, 14.8520^{\circ} \mathrm{E}$, on bark of Acer platanoides, leg., det. M. Smoczyk, 1.07.2015 (POZG); ATMOS Da-15: W Poland, Lubuskie Lakeland, Torzymska Plain, $0.6 \mathrm{~km}$ south-west from the church in the village of Mierczany, forest section 232a of the Torzym Forest Inspectorate, $52.2793^{\circ} \mathrm{N}, 14.9219^{\circ} \mathrm{E}$, on bark of Quercus robur, leg., det. M. Smoczyk, 18.01.2015 (POZG); by the road $0.3 \mathrm{~km}$ southwest from the Gądków Wielki railway station, forest section $117 \mathrm{~d}$ of the Torzym Forest Inspectorate, $52.2436^{\circ} \mathrm{N}, 14.9619^{\circ} \mathrm{E}$, trunk base of Quercus robur, leg., det. M. Smoczyk, 18.01.2015 (POZG); ATMOS Da-27: W Poland, Lubuskie Lakeland, Gryżyński Landscape Park, ca $1.3 \mathrm{~km}$ west of the village of Gryżyna, forest section 285n of the Bytnica Forest Inspectorate, $52.1845^{\circ} \mathrm{N}, 15.2631^{\circ} \mathrm{E}$, on bark of Populus sp., not. M. Smoczyk, 22.04.2016; ATMOS Da-37: W Poland, Lubuskie Lakeland, Gryżyński Landscape Park, $1.8 \mathrm{~km}$ south of the church in the village of Grabin, near Jezioro Jatnik Lake, $52.1092^{\circ} \mathrm{N}, 15.2843^{\circ} \mathrm{E}$, on bark of Padus avium, not. M. Smoczyk, 23.04.2016.

The liverwort Frullania dilatata is widespread in Poland. According to the literature, $F$. dilatata has been found throughout Poland (SzweYKowski \& Koźlicka 1977, SzweYkowski 2006). From the Pojezierze Lubuskie, $F$ dilatata was mentioned in old floras as "frequent" (WARNSTORF 1885, TorKa 1904). In the Pojezierze Lubuskie region, $F$. dilatata has recently been found at numerous localities, but the populations are usually of small size. In Pojezierze Lubuskie, F. dilatata often grows on the bark of various species of deciduous trees and shrubs with subneutral bark reactions (e.g., Padus avium and Acer platanoides), but has also been found growing on trees with more acidic bark, especially Alnus glutinosa and Betula pendula. Frullania dilatata mostly grows in epiphytic bryophyte vegetation in communities from the Orthotrichetalia Hadač in Klika et Hadač 1944 order (class Frullanio dilatatae-Leucodontetea sciuroidis Mohan 1978), and has rarely been noted on trees on the forest edges in nitrophilic communities from the alliance Xanthorion parietinae Ochsner 1928 (class Physcietea Tomaselli \& De Micheli 1952).

\section{Hamatocaulis vernicosus (Mitt.) Hedenäs}

Author: P. PAwLIKOwSKI

ATMOS Bf-12: NE Poland, Masurian Lake District (Pojezierze Mazurskie), Warmia-Masuria Province, Giżycko County, Kruklanki commune, SE from the Borecka Forest (Puszcza Borecka), SW of the village of Możdżany, $54.07287^{\circ} \mathrm{N}, 22.00658^{\circ} \mathrm{E}$, small minerotrophic fen surrounded by agricultural land, desiccated due to intensive drainage by newly renovated drainage ditch, a few small patches (covering a to- 
tal area of ca. 0,5 $\mathrm{m}^{2}$ ) in a deteriorating sedge-moss vegetation overgrowing with trees and shrubs, along with Sphagnum teres and Calliergonella cuspidata, not. P. Pawlikowski, 10.08.2013.

Hamatocaulis vernicosus is a species occurring primarily in very wet rich fens (DIERSSEN 2001, SzCZEPAŃSKI 2010), listed in Annex II of the European Union Habitat Directive (European Directive 92/43/ EEC - Council... 1992). Hamatocaulis vernicosus occurs throughout Poland and, apart from the young post-glacial landscape of northern Poland, the species is threatened with extinction (Stebel 2012). In the Warmia and Masuria Province, its distribution is patchy, but was previously locally abundant (Łachacz \& Olesiński 2000, Łachacz \& Pisarek 2002, SZCZEPAŃSKI 2010, W. Pisarek unpubl. from the years 1995-2016, M. Szczepański unpubl. 2000-2016). In the Borecka Forest (a microregion within the Ełk Lakeland), H. vernicosus was recorded in three mires in the south-eastern part of the forest complex (KOPPE \& Koppe 1931, 1937).

\section{Harpanthus flotovianus (Nees) Nees}

Author: H. Klama

ATMOS Gd-69: S Poland, Western Tatra Mts, Dolina Pyszniańska valley, Babi Potok stream, below Żleb Babie Nogi gully, alt. $1320 \mathrm{~m}$ a.s.l., on wet soil on the escarp of the stream, leg., det. H. Klama, 7.09.1984, ster. (herb. H. Klama).

Harpanthus flotovianus is a subalpine-alpine species that is very rarely found in Poland. Harpanthus flotovianus has been reported in the Sudetes (Karkonosze and Góry Izerskie Mts), Tatra Mountains, Beskid Żywiecko-Orawski Mountains and Gorce Mountains (Klama 1996, MierzeńsKa 1994, SzWeYKowski 2006). Some relict populations of $H$. flotovianus have been reported in the northern Polish lowland (SzWEYKOwsKI 2006). In the Polish part of the Tatra Mountains, four populations of $H$. flotovianus were recorded in the western parts (GóRSKI \& VÁŇA 2014). Harpanthus flotovianus grows in wet habitats, mainly along streams.

\section{Pohlia ludwigii (Spreng. ex Schwägr.) Broth.}

Authors: P. GóRSKI, A. RusińsKA

Slovakia, Western Tatra Mts: 34UDV0453, Roháčska dolina valley, glacial cirque below Mt Brestová (from the north side), alt. $1755 \mathrm{~m}$ a.s.l., Pohlietum ludwigii oligotrichetosum hercynici, leg. P. Górski, 23.07.2006, det. A. Rusińska (KRAM, POZNB); 34UDV0550: Spálená dolina valley, debris slope below rocky walls descending from Mt Baníkov, alt. 1920 m a.s.l., Pohlietum ludwigii oligotrichetosum hercynici, leg. P. Górski, 19.07.2006, det. A. Rusińska (KRAM, POZNB); Spálená dolina valley, debris slope below rocky walls descending from Mt Baníkov, alt. $1875 \mathrm{~m}$ a.s.l., Polytrichetum sexangularis typicum, leg.
P. Górski, 19.07.2006, det. A. Rusińska (POZNB); 34UDV0751, Spálená dolina valley, rocky outcrops near route, above Rázcestie k Roháčskym Plesám, alt. $1530 \mathrm{~m}$ a.s.l., Moerckietum blyttii typicum, leg. P. Górski, 24.07.2006, det. A. Rusińska (POZNB); SLOVAKIA, High TAtra Mts: 34UDV2846: Nefcerka valley, the uppermost glacial cirque, $49.16905^{\circ} \mathrm{N}, 20.02283^{\circ} \mathrm{E}$, alt. $2150 \mathrm{~m}$ a.s.l., Pohlietum drummondii typicum, leg. P. Górski, 3.08.2011, det. A. Rusińska (POZNB), Nefcerka valley, the uppermost glacial cirque, $49.16972^{\circ} \mathrm{N}, 20.02208^{\circ} \mathrm{E}$, alt. $2155 \mathrm{~m}$ a.s.l., Pohlietum ludwigii typicum, leg. P. Górski, 3.08.2011, det. A. Rusińska (KRAM, POZNB); 34UDV3046, Mlynická dolina valley, blocks of rock $S$ from Vyšné Kozie pleso lake, $49.16687^{\circ} \mathrm{N}, 20.04647^{\circ} \mathrm{E}$, alt. $2055 \mathrm{~m}$ a.s.l., Pohlio nutantis-Fuscocephaloziopsietum albescentis, leg. P. Górski, 12.08.2011, det. A. Rusińska (POZNB); 34UDV3047, Hlinská dolina valley, below Výsné Kôprovské sedlo pass, alt. 1950 m a.s.l., comm. with Anthelia juratzkana, leg. P. Górski, 21.08.2006, det. A. Rusińska (POZNB); 34UDV3148, Hincova dolina valley, rocky outcrops near N shore of Vel'ké Hincovo pleso lake, alt. $1940 \mathrm{~m}$ a.s.l., Polytrichetum sexangularis fuscocephaloziopsietosum albescentis, leg. P. Górski, 12.08.2008, det. A. Rusińska (POZNB); 34UDV3349, Žabia Bielovodská dolina valley, blocks of rock near S shore of Nižné Žabie Bielovodské pleso lake, alt. $1681 \mathrm{~m}$ a.s.l., comm. with Fuscocephaloziopsis albescens, leg. P. Górski, 14.08.2009, det. A. Rusińska (KRAM, POZNB); 34UDV3445, Zlomisková dolina valley, Kotlina Lodového plesa basin, rocky walls above E shore of Lodové pleso lake, $49.16292^{\circ} \mathrm{N}$, $20.10985^{\circ}$ E, alt. $2010 \mathrm{~m}$ a.s.1., Pohlietum ludwigii oligotrichetosum hercynici, leg. P. Górski, 6.08.2010, det. A. Rusińska (KRAM, POZNB); 34UDV3446, Rumanova dolinka valley, near NE shore of Vyšné Rumanovo pliesko lake, $49.17205^{\circ} \mathrm{N}, 20.10078^{\circ} \mathrm{E}$, alt. $2135 \mathrm{~m}$ a.s.l., Pohlietum drummondii typicum, leg. P. Górski, 9.08.2011, det. A. Rusińska (POZNB); 34UDV3447, Ťažká dolina valley, upper part of lower glacial cirque, blocks of rock below Mt Pustá stráž, alt. $1825 \mathrm{~m}$ a.s.l., Pohlietum ludwigii oligotrichetosum hercynici, leg. P. Górski, 26.08.2009, det. A. Rusińska (KRAM, POZNB); 34UDV3448: Žabia Bielovodská dolina valley, $\mathrm{W}$ from Mlynárovo sedlo pass, debris slope below rocky walls, $49.18895^{\circ} \mathrm{N}, 20.09562^{\circ} \mathrm{E}$, $49.18917^{\circ} \mathrm{N}, 20.0946^{\circ} \mathrm{E}, \quad 49.18918^{\circ} \mathrm{N}, \quad 20.09477^{\circ} \mathrm{E}$, alt. 1900 m, 1920 m, 1925 m a.s.l., Pohlietum ludwigii polytrichastretosum sexangularis, Polytrichetum sexangularis fuscocephaloziopsietosum albescentis, Pohlietum ludwigii typicum, leg. P. Górski, 4.08.2011, det. A. Rusińska (KRAM, POZNB), Žabia Bielovodská dolina valley, debris slope below rocky walls descending from a ridge Mlynárovo sedlo-Mt Velký Žabi štít, alt. $1885 \mathrm{~m}$ a.s.l., Pohlietum ludwigii typicum, leg. P. Górski, 19.08.2009, det. A. Rusińska (KRAM, POZNB); 34UDV3449: Žabia Bielovodská dolina valley, E from S shore of Nižné Žabie Bielovodské pleso lake, be- 
low rocky walls descending from Mt Prostredný Mlynár, $49.19708^{\circ} \mathrm{N}, 20.09672^{\circ} \mathrm{E}$, alt. $1725 \mathrm{~m}, 1735$ $\mathrm{m}$ a.s.l., Pohlietum ludwigii typicum, Andreaeetum nivalis anthelietosum juratzkanae, leg. P. Górski, 16.08.2009, 4.08.2011, det. A. Rusińska (KRAM, POZNB), Žabia Bielovodská dolina valley, ebris slope below rocky walls descending from a ridge Mlynárovo sedlo-Mt Velký Žabi štít, alt. 1885 m, 1920 m a.s.l., Pohlietum ludwigii typicum, leg. P. Górski, 18.08.2009, det. A. Rusińska (KRAM, POZNB), Žabia Bielovodská dolina valley, rocky walls NW from Mlynárovo sedlo pass, alt. 1920 m a.s.l., comm. with Marsupella sphacelata, Pohlietum ludwigii polytrichastretosum sexangularis, leg. P. Górski, 16.08.2009, det. A. Rusińska (POZNB); 34UDV3546: Kačacia dolina valley, above and E from Zelené pleso Kačacie lake, below sub-glacially eroded step of the upper glacial cirque, $49.17213^{\circ} \mathrm{N}$, $20.11865^{\circ} \mathrm{E}$, alt. $1725 \mathrm{~m}$ a.s.l., Pohlietum ludwigii oligotrichetosum hercynici, leg. P. Górski, 29.08.2012, det. A. Rusińska (KRAM, POZNB); 34UDV3547: Kačacia dolina valley, below rocky walls descending from $\mathrm{Mt}$ Ganek, alt. 1665 m a.s.l., Pohlietum ludwigii polytrichastretosum sexangularis, leg. P. Górski, 22.08.2007, det. A. Rusińska (KRAM, POZNB), Kačacia dolina valley, in a gully descending from Mt Zlobivá, alt. $1745 \mathrm{~m}$ a.s.l., Pohlietum ludwigii polytrichastretosum sexangularis, leg. P. Górski, 22.08.2007, det. A. Rusińska (KRAM, POZNB); 34UDV3548, Ťažká dolina valley, upper part of the lower glacial cirque, blocks of rock below Mt Pustá stráž, alt. 1810 m, 1815 m a.s.l., Pohlietum ludwigii typicum, Luzuletum alpino-pilosae, leg. P. Górski, 24.08.2009, 26.08.2009, det. A. Rusińska (KRAM, POZNB); 34UDV3645, Batizovská dolina valley, Niżnia Batyżowiecka Rówień, near Batizovská skúš$\mathrm{ka}, 49.16088^{\circ} \mathrm{N}, 20.12863^{\circ} \mathrm{E}, 49.1615^{\circ} \mathrm{N}, 20.12965^{\circ} \mathrm{E}$, $49.15992^{\circ} \mathrm{N}, 20.12762^{\circ} \mathrm{E}$, alt. $2135 \mathrm{~m}, 2175 \mathrm{~m}, 2165$ $\mathrm{m}$ a.s.l., Pohlietum ludwigii typicum, comm. with Marsupella sphacelata, leg. P. Górski, 2.08.2010, det. A. Rusińska (KRAM, POZNB); 34UDV3647, Litvorová dolina valley, below rocky walls descending from $\mathrm{Mt}$ Litvorový štít, alt. 1835 m a.s.l., Nardietum scalaris kiaerietosum starkei, leg. P. Górski, 15.09.2009, det. A. Rusińska (POZNB); 34UDV3747, Svištová (Bielovodská) dolina valley, Zmarznutý kotol basin, alt. $2065 \mathrm{~m}$ a.s.l., Polytrichetum sexangularis fuscocephaloziopsietosum albescentis, leg. P. Górski, 1.08.2006, det. A. Rusińska (POZNB); 34UDV3948, Zadná Javorová dolina valley, glacial cirque below Mt Javorový štít, above Žabie Javorové pleso lake, alt. 1970 m a.s.l., Pohlietum ludwigii polytrichastretosum sexangularis, leg. P. Górski, 16.08.2006, det. A. Rusińska (KRAM, POZNB); 34UDV3950, Suchá (Javorová) dolina valley, alt. 1835 m a.s.l., Nardietum scalaris kiaerietosum starkei, leg. P. Górski, 18.08.2006, det. A. Rusińska (KRAM, POZNB); 34UDV4048, Malá Studená dolina valley, Dolina pod Sedielkom, below rocky walls descending from Priečne sedlo pass, alt. $2120 \mathrm{~m}$ a.s.l., Pohlietum ludwigii polytrichastretosum sexangularis, leg. P. Górski, 21.08.2007, det. A. Rusińska (KRAM, POZNB); 34UDV4050, Čierna Javorová dolina valley, Čierna záhrada, rocky walls at the mouth of Ladova dolinka valley, $49.20685^{\circ} \mathrm{N}, 20.17762^{\circ} \mathrm{E}$, alt. 1660 $\mathrm{m}$ a.s.1., Nardietum scalaris kiaerietosum starkei, leg. P. Górski, 25.08.2010, det. A. Rusińska (POZNB); 34UDV4149: Kotlina Piatich Spišských Plies valley, near NE shore of Vyšné Spišské pleso lake, $49.19468^{\circ} \mathrm{N}, 20.19688^{\circ} \mathrm{E}$, alt. $2025 \mathrm{~m}$ a.s.l., comm. with Nardia breidleri, leg. P. Górski, 11.08.2011, det. A. Rusińska (POZNB), Kotlina Piatich Spišských Plies valley, above and NWW from NW shore of Prostredné Spišské pleso lake, $49.193^{\circ} \mathrm{N}, 20.19802^{\circ} \mathrm{E}$, alt. 2025 m a.s.l., comm. with Nardia breidleri, leg. P. Górski, 11.08.2011, det. A. Rusińska (POZNB), Kotlina Piatich Spišských Plies valley, above W shore of Prostredné Spišské pleso lake, alt. 2005 m a.s.l., Pohlietum ludwigii polytrichastretosum sexangularis, leg. P. Górski, 27.08.2007, det. A. Rusińska (KRAM, POZNB).

Poland, Western Tatra Mts: 34UDV1150, Dolina Jarząbcza valley, below rocky walls descending from Niska Przełęcz pass, alt. 1730 m a.s.l., Pohlietum ludwigii oligotrichetosum hercynici, leg. P. Górski, 9.08.2004, det. A. Rusińska (POZNB); 34UDV1251: Dolina Jarząbcza valley, NW slope of Mt Kończysty Wierch, alt. 1720 m, 1735 m a.s.l., Luzuletum alpinopilosae pellietosum neesianae, Nardietum scalaris kiaerietosum starkei, leg. P. Górski, 22.07.2009, det. A. Rusińska (POZNB), Dolina Jarząbcza valley, NW slope of Mt Kończysty Wierch (Jarząbczy Kopieniec), alt. 1720 $\mathrm{m}$ a.s.l., Pohlietum ludwigii oligotrichetosum hercynici, leg. P. Górski, 22.07.2009, det. A. Rusińska (KRAM, POZNB); 34UDV1450: Dolina Starorobociańska valley, E from Krzywy Żleb gully, alt. 1665 m, 1775 $\mathrm{m}$ a.s.l., Luzuletum alpino-pilosae pellietosum neesianae, Pohlietum ludwigii typicum, leg. P. Górski, 23.07.2009, det. A. Rusińska (KRAM, POZNB), Dolina Starorobociańska valley, Zadnie Zagony, Krzywy Żleb gully, alt. $1655 \mathrm{~m}$ a.s.l., Pohlietum ludwigii polytrichastretosum sexangularis, leg. P. Górski, 23.07.2009, det. A. Rusińska (POZNB), Dolina Starorobociańska valley, below rocky walls of main ridge, E from Krzywy Żleb gully, alt. $1775 \mathrm{~m}$ a.s.l., Pohlietum ludwigii polytrichastretosum sexangularis, leg. P. Górski, 23.07.2009, det. A. Rusińska (KRAM, POZNB); 34UDV1649, Kamenistá dolina valley, in the kettle below ridge Mt Blyšt - Pyšne sedlo pass, $49.19465^{\circ} \mathrm{N}, 19.85138^{\circ} \mathrm{E}$, alt. $1820 \mathrm{~m}$ a.s.l., Polytrichetum sexangularis typicum, leg. P. Górski, 25.08.2011, det. A. Rusińska (POZNB); 34UDV2752: Dolina Gąsienicowa valley, glacial cirque below Mt Świnica, above Czerwone Stawki lakes, Grzędy, alt. 1875 m a.s.l., Pohlietum ludwigii oligotrichetosum hercynici, leg. P. Górski, 20.08.2005, det. A. Rusińska (KRAM, POZNB), Dolina Gąsienicowa valley, Zadnie Koło, blocks of rock, near small lake, $49.22333^{\circ} \mathrm{N}, 20.00803^{\circ} \mathrm{E}$, alt. $1877 \mathrm{~m}$ a.s.l., Pohlietum ludwigii polytrichastretosum sexangularis, leg. P. Górski, 30.08.2011, det. A. Rusińska (POZNB); 
Poland, High Tatra Mts: 34UDV2850, Dolina Pięciu Stawów Polskich valley, below rocky walls descending from Mt Kotelnica, $49.2038^{\circ} \mathrm{N}, 20.01873^{\circ} \mathrm{E}$, alt. $1875 \mathrm{~m}$ a.s.l., Pohlietum ludwigii oligotrichetosum hercynici, leg. P. Górski, 23.08.2012, det. A. Rusińska (KRAM, POZNB); 34UDV2852: Dolina Gąsienicowa valley, below Zmarzła Przełęcz pass, $49.22067^{\circ} \mathrm{N}$, $20.02277^{\circ} \mathrm{E}, 49.22067^{\circ} \mathrm{N}, 20.02277^{\circ} \mathrm{E}$, alt. $1990 \mathrm{~m}$ a.s.l., Andreaeetum nivalis anthelietosum juratzkanae, Pohlietum ludwigii polytrichastretosum sexangularis, leg. P. Górski, 8.08.2012, det. A. Rusińska (POZNB), Dolina Gąsienicowa valley, SW from Zmarzły Staw lake, a stony gully, $49.22277^{\circ} \mathrm{N}, 20.02103^{\circ} \mathrm{E}$, alt. $1865 \mathrm{~m}$ a.s.l., Pohlietum ludwigii polytrichastretosum sexangularis, leg. P. Górski, 31.08.2011, det. A. Rusińska (POZNB); 34UDV2950, Dolina Pięciu Stawów Polskich valley, below rocky walls descending from Mt Wyżni Liptowski Kostur, alt. 2040 m a.s.l., Pohlietum ludwigii polytrichastretosum sexangularis, leg. P. Górski, 25.07.2005, det. A. Rusińska (KRAM, POZNB); 34UDV3049: Dolina za Mnichem valley, below Wrota Chałubińskiego pass, $49.19255^{\circ} \mathrm{N}, 20.04687^{\circ} \mathrm{E}$, alt. $1955 \mathrm{~m}$ a.s.l., Pohlietum ludwigii typicum, leg. P. Górski, 26.08.2012, det. A. Rusińska (KRAM, POZNB), Dolina za Mnichem valley, between Wyżni Staw Staszica lake and Wyżnie Mnichowe Stawki lakes, $49.19175^{\circ} \mathrm{N}$, $20.0485^{\circ} \mathrm{E}, 49.19183^{\circ} \mathrm{N}, 20.04947^{\circ} \mathrm{E}$, alt. $1970 \mathrm{~m}$ a.s.l., Pohlietum ludwigii typicum, Polytrichetum sexangularis fuscocephaloziopsietosum albescentis, leg. P. Górski, 26.08.2012, det. A. Rusińska (KRAM, POZNB); 34UDV3149, Dolina za Mnichem valley, rocky outcrops above Stawki Staszica lakes, alt. 1835 m a.s.l., Bucklandiello sudetici-Marsupelletum emarginatae, leg. P. Górski, 20.09.2005, det. A. Rusińska (POZNB); 34UDV3151, Dolina Pięciu Stawów Polskich valley, below rocky walls descending from Marchwiczna Przełęcz pass, alt. $1900 \mathrm{~m}$ a.s.l., Polytrichetum sexangularis fuscocephaloziopsietosum albescentis, leg. P. Górski, 26.07.2005, det. A. Rusińska (POZNB); 34UDV3248, Dolina Rybiego Potoku valley, rocky outcrops above Czarny Staw pod Rysami lake, alt. $1715 \mathrm{~m}$ a.s.l., Nardietum scalaris kiaerietosum starkei, leg. P. Górski, 18.08.2004, det. A. Rusińska (POZNB).

Pohlia ludwigii is an arctic-alpine species occurring on wet and sandy soils, most often found in late snow fields. In Poland, P. ludwigii has been reported in the Tatra and Karkonosze Mountains (see references in GórsKi 2015). From personal studies conducted by the first author between 2002 and 2014, $P$. ludwigii was identified as a common species in the Polish and Slovakian Tatra Mountains (in Slovakia, $P$. ludwigii is categorized as NT-endangerment; KUBINSKÁ et al. 2001). This study presents another 67 localities of this plant from the High and Western Tatra Mountains. In this massive, Pohlia ludwigii creates scanty-species phytocoenoses described as Pohlietum ludwigii (BALCERKIEWICZ 1984, GóRSKI 2015, 2016). In the Tatra Mountains, Pohlia ludwigii has been found within the altitude range of $1,530-2,310 \mathrm{~m}$ a.s.l. In studies carried out between 2011 and 2013 by P. Górski, Pohlietum ludwigii phytoceonoses could be found during snow melts in June and the first half of July.

\section{Riccardia latifrons (Lindb.) Lindb.}

Authors: H. Klama, A. Salachna

ATMOS Gd-33: S Poland, Beskid Żywiecko-Orawski Mts, Wielka Racza group (Grupa Wielkiej Raczy), Silesian Province, Żywiec County, Soblówka village, valley of Cichy potok stream, $49.42389^{\circ} \mathrm{N}, 19.13556^{\circ} \mathrm{E}$, alt. $700 \mathrm{~m}$ a.s.l., on decaying wood of tree stump, leg., det. H. Klama, A. Salachna, 23.09.2011, ster. (herb. H. Klama).

Riccardia latifrons is an epixylic liverwort found in lowland-mountain regions of Poland. In the mountains, $R$. latifrons grows on rotten wood in the forest lower belt. Riccardia latifrons is a very rare species in the Beskidy Zachodnie Mountains, and has been recorded in only a few instances in the Beskid Śląski Mountains (Rejment-GrochowsKa 1950), Beskid Żywiecko-Orawski Mountains (KLAmA 1996), Gorce Mountains (MendelaK 1977, MierzeńsKa 1994) and Beskid Sądecki Mountains (Mamczarz 1977, MenDELAK 1977, SZWEyKOWSKI \& KoźLICKA 1980). The liverwort is also rarely found in the Tatra Mountains (SZWEeYKowsKi \& Klama 2010, Górski \& VÁŇA 2014).

\section{Scorpidium scorpioides (Hedw.) Limpr.}

Author: P. PAwLIKowsKi

ATMOS Bf-34: NE Poland, Masurian Lake District, Ełk Lakeland (Pojezierze Ełckie), Warmia-Masuria Province, Ełk County, Stare Juchy, mire bordering Łaśmiady lake SW of the village of Sikory Juskie, $53.90281^{\circ} \mathrm{N}, 22.26146^{\circ} \mathrm{E}$, large, topogenous, temporarily inundated extremely rich fen developed in the overgrown part of the lake, dominating and forming extensive carpets covering the area of over 3 hectares, along with Campylium stellatum, Limprichtia cossonii, Carex elata and C. lasiocarpa, not. P. Pawlikowski 2006-2016, leg., det. P. Pawlikowski, 09.2009 (WA).

Scorpidium scorpioides is a prominent component of rich fen vegetation in the boreal zone of the Northern Hemisphere. Scorpidium scorpioides grows in temporarily or continuously inundated places within rich fens, including waterlogged carpets, small pools, and even shallow lake bottoms (Ochyra et al. 1988a). In Poland, S. scorpioides is considered endangered (category E, ŻARNOWIEC et al. 2004). Scorpidium scorpioides has been recorded throughout the country, but is mainly aggregated in the post-glacial landscapes of northern Poland and in some upland areas (e.g., the Polesie region in eastern Poland) (Ochyra et al. 1988a). Scorpidium scorpioides is also found in north-eastern Poland, sometimes abundantly (locally in the East Suwałki Lakeland and Augustów Plain, e.g., JuTRzEN- 
Ka-Trzebiatowski \& SzarejKo 2001, Pawlikowski et al. 2009, PAwLIKowsKi 2010a, SokoŁowski 2010). However, $S$. scorpioides has never been frequently recorded in the Warmia and Masuria Province (OchYra et al. 1988a). Although S. scorpioides had been reported in the eastern part of this region (Koppe \& Koppe 1931, BLOCH et al. 1979), it is now rarely found there and its numbers are decreasing, with only a few recent published records (e.g., in "Jeziorko koło Drozdowa" nature reserve) (ŁACHACZ \& OlEsIŃsKi 2000; P. Pawlikowski unpubl. 2012, 2016) and in the famous mire near the village of Zocie (Bloch-OrłowsKa \& PisAreK 2005; P. Pawlikowski unpubl. 2009, 2013).

\section{Sphagnum fuscum (Schimp.) H. Klinggr.}

\section{Author: P. PAwLIKOWSKI}

ATMOS Cd-97: Central Poland, Toruń-Eberswalde Urstromtal (Pradolina Toruńsko-Eberswaldzka), Płock Basin (Kotlina Płocka), Mazovia Province, Płock County, Płock commune, Gostynin-Włocławek Landscape Park, 'Jastrząbek' nature reserve, $52.50385^{\circ} \mathrm{N}$, $19.60738^{\circ} \mathrm{E}$, minerotrophic mire bordering SE part of the small, vanishing Jeziorko lake, single hummock in the Sphagnum-dominated fen with Betula pubescens tree stand, along with Sphagnum palustre, S. teres, Phragmites australis, Thelypteris palustris, Oxycoccus palustris and Carex acutiformis, leg., det. P. Pawlikowski, 6.08.2016 (WA).

Sphagnum fuscum is a mountain-arctic-oceanicsubcontinental species and an important component of the moss layer of raised bogs, occurring also in some fens (DiERssen 2001) and indicating habitat changes toward ombrotrophic conditions (ĆwIKLINSKA 2007). The rapid vanishing of $S$. fuscum from acidic mires in the region of Pomerania, where it used to be common, has been documented (JASNOwSKI et al. 1968) and, generally, the species is decreasing in its southern lowland European range (DiERSSEN 2001). Sphagnum fuscum is considered vulnerable in Poland (category V, ŻARNOwIEC et al. 2004). Sphagnum fuscum has been recorded in the northern and western lowland areas of Poland, as well as in the mountains (SzAFRAN 1957), and remains only locally frequent in selected lakeland regions of the post-glacial landscapes of northern Poland (e.g., Karczmarz \& SoKo£OWSKI 1985, Herbichowa et al. 2007, PAWLiKowski 2010b, P. Pawlikowski unpubl. 2003-2016) and submountain raised bogs of the Orawa-Nowy Targ Basin (Koczur 2007). In the predominating lowland landscapes of central Poland, where the newly discovered locality is situated, S. fuscum has rarely been recorded, with only two records to date in the Mazovia Province: one in the Torfowisko Serafin nature reserve (WĄs 1960, P. Pawlikowski unpubl. 2013-2016) and one in the Zwoleńka river valley near the village of Stara Siekierka (JarzombKowski \& KozUB 2011).

\section{Tomentypnum nitens (Hedw.) Loeske}

Author: P. PawlikowsKi

ATMOS Bf-12: NE Poland, Masurian Lake District, Warmia-Masuria Province, Giżycko County, Kruklanki commune, SE of the Borecka Forest, SW of the Możdżany village, $54.07287^{\circ} \mathrm{N}, 22.00658^{\circ} \mathrm{E}$, small minerotrophic fen surrounded by agricultural land, desiccated due to intensive drainage by newly renovated drainage ditch, small hummock in a deteriorating sedge-moss vegetation overgrowing with trees and shrubs, along with Sphagnum teres and Calliergonella cuspidata, not. P. Pawlikowski, 10.08.2013.

Tomentypnum nitens is considered vulnerable in Poland (category V, ŻARNOWIEC et al. 2004) but remains relatively common in minerotrophic fens in some region, including some lakeland areas of northern and north-western Poland, the Biebrza area, uplands of southern Poland, and the Carpathians (Ochyra et al. 1988b). In the Warmia and Masuria Province, the T. nitens distribution is patchy and recent published records are rather limited (ŁACHACZ \& OLESIŃSKI 2000, Łachacz \& Pisarek 2002, 2007, Bloch-OrŁowska \& Pisarek 2005, Pawlikowski \& JarzombKowski 2010), although the species seems more numerous in the eastern part of the region (BLOCH et al. 1979). It should be noted that many existing localities remain unpublished (M. Szczepański unpubl., W. Pisarek unpubl. from the years 1995-2016). In the Borecka Forest (a microregion within the Ełk Lakeland), the species was recorded only by KopPe \& KopPe (1937, see Ochyra et al. 1988b) from two sites: (presently degraded) peatland between the village of Jabłonowo and Ciche lake, and peatland situated west of the northernmost part of Szwałk Wielki lake.

\section{REVISIONS}

1. Hymenostylium recurvirostrum (Hedw.) Dixon published in Fојсік (1999), page 95 [ATMOS Ed-61: S Poland, Wieluńska Upland (Wyżyna Wieluńska), Śląskie Province, Rębielice Królewskie, $50.98855^{\circ} \mathrm{N}$, $18.84386^{\circ} \mathrm{E}$, shaded crevice of limenstone on a hill, leg., det. B. Fojcik, 8.05.1995] - est Eucladium verticillatum (Hedw. ex Brid.) Bruch \& Schimp., rev. B. Fojcik, 2016 (KTU).

\section{REFERENCES}

BalCERKIEWICZ S. (1984): Roślinność wysokogórska Doliny Pięciu Stawów Polskich w Tatrach i jej przemiany antropogeniczne. Uniwersytet im. Adama Mickiewicza, Seria Biologia 25: 1-191.

Bloch M., Karczmarz K., SokoŁowski A. W. (1979): Nowe dane do flory mszaków północno-wschodniej Polski. 2. Annales Universitatis Mariae Curie-Skłodowska, Sectio C, Biologia 34(5): 47-53. 
Bloch-Orfowska J., Pisarek W. (2005): Rzadkie i zagrożone rośliny naczyniowe oraz mchy torfowiska "Zocie" na Pojezierzu Ełckim. Chrońmy Przyrodę Ojczystą 61(3): 5-12.

ĆWIKLINSKA P. (2007): Specyfika ekologiczna kęp z udziałem Sphagnum fuscum na torfowiskach minerogenicznych. Acta Botanica Cassubica 6: 131140.

Dierssen K. (2001): Distribution, ecological amplitude and phytosociological characterization of European bryophytes. Bryophytorum Bibliotheca 56: 1-289.

DudA J., VÁŇA J. (1985): Rozšíření játrovek v Československu. Vol. 42. Časopis Slezského Muzea, Series A, Opava 34: 1-20.

Düll R. (1984): Distribution of the European and Macaronesian mosses (Bryophytina). Part 1. Bryologische Beiträge 4: 1-109.

FoJcIK B. (1999): Mosses of the Wielun Upland (Southern Poland). Fragmenta Floristica et Geobotanica 44(1): 77-128.

Fојсік B. (2011): Distribution atlas of mosses of the Cracow-Częstochowa Upland. Centrum Dziedzictwa Przyrody Górnego Śląska, Katowice.

GóRsKi P. (2015): 15. Pohlia ludwigii (Spreng. ex Schwägr.) Broth. In: P. Górski, A. Rusińska (eds). New distributional data on bryophytes of Poland and Slovakia, 3. Steciana 19(3): 167-168.

GóRSKI P. (2016): Snowbed bryophyte vegetation of the Tatra Mountains (Western Carpathians, Poland and Slovakia). Nova Hedwigia 102 (1-2): 9-67.

GÓRSKI P., VÁŇA J. (2014): A synopsis of liverworts occurring in the Tatra Mountains (Western Carpathians, Poland and Slovakia): checklist, distribution and new data. Preslia 86 (4): 381-485.

Herbichowa M., Pawlaczyk P., Stańko R. (2007): Ochrona wysokich torfowisk bałtyckich na Pomorzu. Wydawnictwo Klubu Przyrodników, Warszawa.

JarzombKowski F., Kozub Ł. (2011): Stan zagrożenia i ochrony mechowisk w krajobrazie rolniczym Mazowsza. In: W. Dembek, A. Gutkowski, H. Piórkowski (eds). Współczesne narzędzia identyfikacji oraz ochrony mokradeł i muraw w krajobrazie rolniczym. Instytut Technologiczno-Przyrodniczy, Zakład Ochrony Przyrody i Krajobrazu Wiejskiego, Falenty: 85-105.

JASNOWSKI M., JASNOWSKA J., MARKOWSKI S. (1968): Ginące torfowiska wysokie i przejściowe w pasie nadbałtyckim Polski. Ochrona Przyrody 33: 69-124.

Jutrzenka-Trzebiatowski A., Szarejko T. (2001): Zespół Caricetum buxbaumii w Wigierskim Parku Narodowym. Fragmenta Floristica et Geobotanica Polonica 8: 149-171.

KarcZMARZ K., SoKoŁowsKi A.W. (1985): Brioflora projektowanego Wigierskiego Parku Narodowego.
Annales Universitatis Mariae Curie-Skłodowska, Sectio C, Biologia 40: 193-213.

Klama H. (1996): Wątrobowce (Hepaticae) Beskidu Żywiecko-Orawskiego (Karpaty Zachodnie). Monographiae Botanicae 79: 1-144.

Koczur A. (2007): Rośliny torfowisk wysokich w Kotlinie Orawsko-Nowotarskiej (Karpaty Zachodnie). 2. Gatunki charakterystyczne dla związku Sphagnion magellanici. Fragmenta Floristica et Geobotanica Polonica 14(1): 91-101.

Koppe F., Koppe K. (1931): Zur Moosflora Ostpreussens. Unser Ostland 1(6): 299-394.

Koppe F., Koppe K. (1937): Zur Moosflora Ostpreussens. 2. Schriften der Physikalisch-Ökonomischen Gesellschaft zu Königsberg 69: 357-382.

Kubinská A., Janovicová K., Šoltés R. (2001): Aktualizovaný zoznam pečeňoviek, rožtekov a machov Slovenska. Bryonora 28: 4-10.

ŁACHACZ A., OlesiŃSKI L. (2000): Flora i roślinność trzęsawiskowego torfowiska Jeziorko na Pojezierzu Mazurskim. Fragmenta Floristica et Geobotanica Polonica 7: 129-144.

Łachacz A., Pisarek W. (2002): Rzadsze gatunki roślin naczyniowych i mchów Nietlickiego Bagna na Pojezierzu Mazurskim. Acta Botanica Warmiae et Masuriae 2: 53-61.

ŁaCHACZ A., PIsAREK W. (2007): Flora mszaków i roślin naczyniowych rezerwatu przyrody Małga na Równinie Mazurskiej. Parki Narodowe i Rezerwaty Przyrody 26(2): 17-28.

Mamczarz H. (1977): Brioflora i zbiorowiska mszaków Beskidu Sądeckiego. Part 1. Brioflora Beskidu Sądeckiego. Monographiae Botanicae 54: 1-158.

MeNDELAK M. (1977): Wyniki rewizji materiałów zielnikowych rodzaju Riccardia Gray z terenu Polski. Fragmenta Floristica et Geobotanica 23(3-4): 409-417.

MierzeŃsKa M. (1994): Wątrobowce Gorców. Fragmenta Floristica et Geobotanica Polonica 1: 235346.

Ochyra R., Szmajda P., Bednarek H., Bocheński W. (1988a): M. 524. Scorpidium scorpioides (Hedw.) Limpr. In: Z. Tobolewski, T. Wojterski (eds). Atlas of the geographical distribution of spore plants in Poland. Vol. 3. Ser. 5. Mosses (Musci). W. Szafer Institute of Botany of the Polish Academy of Sciences, Państwowe Wydawnictwo Naukowe, Warszawa-Poznań: 41-47.

Ochyra R., Szmajda P., Bednarek H., Bocheński W. (1988b): M. 539. Tomentypnum nitens (Hedw.) Limpr. In: Z. Tobolewski, T. Wojterski (eds). Atlas of the geographical distribution of spore plants in Poland. Vol. 3. Ser. 5. Mosses (Musci). W. Szafer Institute of Botany of the Polish Academy of Sciences, Państwowe Wydawnictwo Naukowe, Warszawa-Poznań: 53-61.

Pawlikowski P. (2010a): Torfowiska Pojezierza Sejneńskiego. In: A. Obidziński (ed.). Z Mazowsza 
na Wileńszczyznę. Zróżnicowanie i ochrona szaty roślinnej pogranicza Europy Środkowej i Północno-Wschodniej. Polskie Towarzystwo Botaniczne - Zarząd Główny, Warszawa: 358-380.

PAwLIKowski P. (2010b): Wybrane torfowiska północno-wschodniej Polski. In: A. Obidziński (ed.). Z Mazowsza na Wileńszczyznę. Zróżnicowanie i ochrona szaty roślinnej pogranicza Europy Środkowej i Północno-Wschodniej. Polskie Towarzystwo Botaniczne - Zarząd Główny, Warszawa: 327-407.

Pawlikowski P., JarzombKowski F. (2010): Torfowiska Puszczy Rominckiej. In: A. Obidziński (ed.). Z Mazowsza na Wileńszczyznę. Zróżnicowanie i ochrona szaty roślinnej pogranicza Europy Środkowej i Północno-Wschodniej. Polskie Towarzystwo Botaniczne - Zarząd Główny, Warszawa: 390-407.

Pawlikowski P., Jarzombrowski F., Wołkowycki D., Kozub Ł., Zaniewski P., Bakanowska O., Banasiak Ł., Barańska K., Bielska A., Biereżnoj U., Galus M., Grzybowska M., Kapler A., Karpowicz J., SADOWSKA I., ZARZECKI R. (2009): Rare and threatened plants of the mires in the intensively managed landscape of the Góry Sudawskie region (NE Poland). Roczniki Akademii Rolniczej w Poznaniu 388, Botanika-Steciana 13: 29-36.

Rejment-Grochowska I. (1950): Czynniki ekologiczne i rozmieszczenie geograficzne wątrobowców (Hepaticae) Beskidu Sląskiego. Prace Biologiczne Śląskie 2: 3-71.

RUsiŃSKA A. (1995): New locality of Campylopus flexuosus (Musci, Dicranaceae) in West Pomerania and a review of its distribution in Poland. Fragmenta Floristica et Geobotanica 40(1): 305-309.

Smith A.J.E. (2004): The moss flora of Britain and Ireland. Cambridge University Press, Cambridge.

Sокоєоwsкi A.W. (2010): Puszcza Augustowska. Centrum Informacyjne Lasów Państwowych, Warszawa.

Stebel A. (2006): The mosses of the Beskidy Zachodnie as a paradigm of biological and environmental changes in the flora of the Polish Western Carpathians. Habilitation Thesis 17/2006. Medical University of Silesia in Katowice, Sorus, Katowice-Poznań.

Stebel A. (2007): New distribution data for Campylopus flexuosus (Bryopsida) in Poland. Chrońmy Przyrodę Ojczystą 63(2): 93-98.

Stebel A. (2012): 1393 Haczykowiec błyszczący Hamatocaulis vernicosus (Mitt.) Hedenäs. In: J. Perzanowska (ed.). Monitoring gatunków roślin. Przewodnik metodyczny 2. Główny Inspektorat Ochrony Środowiska, Warszawa: 127-140.

Stebel A. (2015): Contribution to the bryoflora of the Wiśnickie Foothills (Western Carpathians, Poland). Acta Musei Silesiae, Scientiae Naturales 64: 131-139.
Stebel A. (2016): Contribution to the moss flora of the Magura National Park (Western Carpathians, Poland). Fragmenta Naturae 49: 14-26.

Stebel A., Czarnota P. (2012): Wykaz mchów pasma Gorców w polskich Karpatach Zachodnich. Ochrona Beskidów Zachodnich 4: 7-25.

Stebel A., Ochyra R., Vončina G. (2010): Mosses of Pieniny Range (Polish Western Carpathians). Sorus, Poznań.

Stebel A., Zubel R., Fojcik B., Górski P., Rusińska A., SAwicki J., Szczepański M., Wolski G.J. (2011): Bryophytes of the Muncul nature reserve in the Beskid Wysoki Range (Polish Western Carpathians). In: A. Stebel, R. Ochyra (eds). Chorological Studies on Polish Carpathian Bryophytes. Sorus, Poznań: 193-205.

Szafran B. (1957): Mchy (Musci). Vol. 1. Flora Polska. Rośliny zarodnikowe Polski i ziem ościennych. Państwowe Wydawnictwo Naukowe, Warszawa.

SzczepaŃsKi M. (2010): Sierpowiec błyszczący Drepanocladus vernicosus (Mitt.) Warnst. In: Cz. Hołdyński (ed.). Siedliska i gatunki Natura 2000. Raport $z$ inwentaryzacji przyrodniczej przeprowadzonej w lasach Regionalnej Dyrekcji Lasów Państwowych w Olsztynie i części Regionalnej Dyrekcji Lasów Państwowych w Białymstoku w latach 2006-2008. Mantis, Olsztyn: 175-179.

SzWEYKowsKi J. (1960): Materiały do flory wątrobowców Tatr. Prace Komisji Biologicznej Poznańskiego Towarzystwa Przyjaciół Nauk 21(3): 3-92.

SzWEYKowsKi J. (1967): H. 82. Barbilophozia barbata (Schmidel) Loeske. In: J. Czubiński, J. Szweykowski (eds). Atlas of geographical distribution of spore plants in Poland. Ser. 4. Liverworts (Hepaticae). Vol. 4. Polska Akademia Nauk, Instytut Botaniki, Państwowe Wydawnictwo Naukowe, Warszawa, Poznań.

SzWEYKOWSKi J. (2006): An annotated checklist of Polish liverworts. - Krytyczna lista wątrobowców i glewików Polski. Biodiversity of Poland. Vol. 4. W. Szafer Institute of Botany, Polish Academy of Sciences, Kraków.

SzWEYKowsKi J., BuczKowsKa K., OdRzyKoski I. (2005): Conocephalum salebrosum (Marchantiopsida, Conocephalaceae) - a new Holarctic liverwort species. Plant Systematics and Evolution 253: 133-158.

SzWEYKowSKI J., Klama H. (2010): Liverworts of the Tatra National Park. A checklist. W. Szafer Institute of Botany, Polish Academy of Sciences, Kraków.

SzweYkowski J., KoźLICKA M. (1977): H. 242. Frullania dilatata (L.) Dumort. In: J. Szweykowski, T. Wojterski (eds). Atlas of geographical distribution of spore plants in Poland. Ser. 4. Liverworts (Hepaticae). Vol. 9. Polska Akademia Nauk, Instytut Botaniki, Państwowe Wydawnictwo Naukowe, Warszawa, Poznań: 21-23. 
SzWEyKOWSKi J., KoźLICKA M. (1980): H. 44. Riccardia latifrons Lindberg. In: J. Szweykowski, T. Wojterski (eds). Atlas of geographical distribution of sporeplants in Poland. Ser. 4. Liverworts (Hepaticae). Part 10. Państwowe Wydawnictwo Naukowe, Warszawa-Poznań: 5-8.

TORKA V. (1904): Moose des Kreises Zullichau-Schwiebus. Helios 21: 51-86.

WajDa P., Klama H. (2014): Wątrobowce (Marchantiophyta) Cygańskiego Lasu w Bielsku-Białej. In: XVIII Konferencja Naukowo-Techniczna, Zapobieganie zanieczyszczeniu, przekształcaniu i degradacji środowiska, Szczyrk, 20-21 listopada 2014 r. Streszczenia prezentacji. Wydawnictwo Naukowe Akademii Techniczno-Humanistycznej w Bielsku-Białej, Bielsko-Biała: 85 .

WARnstorf C. (1885): Moosflora der Provinz Brandenburg. Eine systematische zusammenstellung der bisher in diesem Gebiete beobachteten Leber-, Torf- und Laubmoose. Verhandlungen des botanischen Vereins der Provinz Brandenburg 27: 1-94.
WĄS S. (1960): Zakładanie się i rozwój warstw mszystych torfu w kompleksie torfowym "Pupkowizna" na Kurpiowszczyźnie. Zeszyty Problemowe Postępów Nauk Rolniczych 25: 79-98.

Żarnowiec J., Stebel A., Ochyra R. (2004): Threatened moss species in the Polish Carpathians in the light of a new Red-list of mosses in Poland. In: A. Stebel, R. Ochyra (eds). Bryological studies in the Western Carpathians. Sorus, Poznań: 9-28.

For citation (1): GóRski P., Vončina G., SMOczYK M., Klama H., Šoltés R., Wilhelm M., RutKowsKa M. (2017): New distributional data on bryophytes of Poland and Slovakia, 9. Steciana 21(1): 31-40. doi: $10.12657 /$ steciana.021.004

For citation (2): KlaMA H. (2017): 8. Harpanthus flotovianus (Nees) Nees. In: P. Górski, A. Rusińska (eds). New distributional data on bryophytes of Poland and Slovakia, 9. Steciana 21(1): 34. doi: 10.12657/steciana.021.024 